Revue des patrimoines

Le cheval et ses patrimoines (1ère partie)

\title{
Conclusions et perspectives ouvertes par le colloque de Tulle
}

Jean-Pierre Digard

\section{OpenEdition}

\section{Journals}

Édition électronique

URL : http://journals.openedition.org/insitu/9652

DOI : 10.4000/insitu.9652

ISSN : 1630-7305

\section{Éditeur}

Ministère de la culture

Référence électronique

Jean-Pierre Digard, «Conclusions et perspectives ouvertes par le colloque de Tulle », In Situ [En ligne], 18 | 2012, mis en ligne le 31 juillet 2012, consulté le 23 avril 2019. URL : http:// journals.openedition.org/insitu/9652 ; DOI : 10.4000/insitu.9652

Ce document a été généré automatiquement le 23 avril 2019

\section{(c) $($ i) $(9)$}

In Situ Revues des patrimoines est mis à disposition selon les termes de la licence Creative Commons Attribution - Pas d'Utilisation Commerciale - Pas de Modification 4.0 International. 


\title{
Conclusions et perspectives ouvertes par le colloque de Tulle
}

\author{
Jean-Pierre Digard
}

1 C'est pour moi un honneur d'avoir à tirer les conclusions d'un colloque qui a été ouvert par cette grande dame de l'élevage et historienne émérite qu'est Nicole de Blomac. Honneur au demeurant quelque peu immérité puisque je suis un chercheur et, donc, un utilisateur beaucoup plus qu'un conservateur d'archives et de collections. Les réactions que je vais livrer ici seront donc en grande partie celles d'un candide. J'ai, durant ma carrière, fréquenté, par nécessité professionnelle, quelquefois aussi par affinités, des archivistes et des conservateurs. Je n'ignore rien des difficultés qui ternissent parfois les relations qu'entretiennent les chercheurs d'une part, et les archivistes et conservateurs d'autre part: les premiers se montrent souvent arrogants, les seconds volontiers possessifs. J'ai découvert à Tulle une troisième catégorie d'acteurs: les pourvoyeurs, donateurs ou services « versants » d'archives et de collections, qui, par négligence ou par mauvaise volonté, posent eux aussi leur lot de difficultés. Ce qui est certain, c'est qu'il ne m'était jamais arrivé de rencontrer, réunis en un même lieu au même moment, un aussi grand nombre d'archivistes et de conservateurs qu'à ce colloque. À cette occasion, j'ai découvert à la fois la difficulté de leur tâche, leur compétence, leur professionnalisme et leur dévouement au service public. J'ai également été frappé par la qualité et la sérénité $\mathrm{du}$ dialogue interdisciplinaire qui s'est établi ici durant ces trois jours. À tous les archivistes et les conservateurs présents à Tulle, je voudrais donc exprimer ma considération et mon respect. Et je ne doute pas, dans ces conditions, que le but de ce premier colloque ait été atteint - but qui était, rappelons-le, de sensibiliser les acteurs du patrimoine au recensement, à la conservation et à la valorisation des patrimoines du cheval.

$2 \mathrm{Si}$, comme dit le dicton, charité bien ordonnée commence par soi-même, alors j'ai été servi au-delà de toute espérance : j'ai beaucoup appris, notamment sur des sites, des collections ou des instruments de travail dont j'ignorais à peu près tout auparavant. J'ai découvert l'ampleur et la diversité des patrimoines du cheval, sans en être vraiment 
surpris tant cet animal était omniprésent en France jusque dans les années 1950. J'ai pris conscience par la même occasion de l'ampleur et de la multiplicité des problèmes liés au recensement et à la conservation de ces patrimoines, dont la récente fusion de l'École nationale d'équitation et des Haras nationaux dans un Institut français du cheval et de l'équitation (IFCE), ainsi que le désolant spectacle du haras de Pompadour à peu près vide de chevaux, sont des amplificateurs et des révélateurs parmi d'autres.

3 J'avais annoncé des questions de candide, sans doute naïves, voire même iconoclastes, en tout cas personnelles, mais dont j'espère qu'elles pourront servir à alimenter le programme de colloques ultérieurs et de nouvelles recherches. Voici ces questions.

La première concerne un phénomène surprenant, omniprésent, mais, semble-t-il, indicible (tout le monde en parle à mots couverts, sans jamais le nommer) : c'est la mystérieuse «évaporation» des archives et des collections mobilières publiques (archives, livres, harnachements et même voitures). Ce sujet sensible devrait, me semble$\mathrm{t}$-il, constituer un objet de recherche à part entière, à la fois pour sa prévention en amont et pour son traitement en aval.

5 Une deuxième question est celle des archives et des collections privées, qui sont trop souvent dispersées (comme le fut la collection Gouraud de selles et harnachements vendue à Drouot en 2005) ou qui peinent à trouver un hébergement satisfaisant (comme la collection Desliens d'instruments vétérinaires). N’y a-t-il rien à faire pour éviter ou limiter ces difficultés?

6 Troisième question, probablement iconoclaste, peut-être même scandaleuse aux yeux de maints conservateurs : doit-on, peut-on tout conserver, tout restaurer? Si non, où se situe la frontière, suivant quels critères ? Pour les savoir-faire relevant de la culture orale ou les patrimoines génétiques, la réponse passe par l'invocation d'un principe de précaution selon lequel il est toujours plus facile de conserver ce qui existe que d'avoir à reconstituer ce qu'on aura laissé disparaître. En revanche, s'agissant des bâtiments et des matériels, il ne parait pas évident de conserver indéfiniment des éléments dont la conservation interdirait toute utilisation ou reconversion.

7 Le plus gros des questions soulevées est plus spécifique au cheval et à ses patrimoines qui, quoi qu'on en dise, ne seront jamais tout à fait des patrimoines comme les autres. D'abord pour la variété des secteurs particuliers concernés, qui n'ont été abordés à Tulle qu'à la marge (chevaux de travail par Gersende Piernas, par exemple) mais qui devront être traités lors des prochains colloques ou ateliers : cavaleries militaires, courses hippiques, cirque, sports et loisirs équestres (que l'on aurait tort de croire limités à l'époque contemporaine), matériels autres que les voitures hippomobiles et harnachements. Sur ce dernier point, une question fort importante a été posée dans la discussion par JeanMichel Leniaud, celle de l'histoire des selles. Certes, nous manquons de séries suffisamment complètes, surtout pour les époques anciennes. Surtout, il faut savoir que l'histoire des selles se réduit à peu près à l'histoire des arçons, qui demeurent inaccessibles sauf à dépiauter les selles qui figurent dans les collections. Une suggestion m'a été faite par Sophie Termignon du musée Au Temps des Calèches: la radiographie. Cette idée justifierait une consultation du C2RMF et, si la faisabilité en était avérée, une campagne de recherche.

8 Autre particularité des activités relatives au cheval : la place considérable qu'y occupent les connaissances éthologiques, les techniques et les manières de faire transmises oralement, voire par simple imitation, de maître à élève ou à apprenti - en équitation, 
écrivait le général L'Hotte, "les livres n'apprennent qu'à ceux qui savent déjà ». La sauvegarde de ces connaissances passe par le recueil ethnographique de la culture orale, et plus sûrement encore par la perpétuation de la pratique; tel est du reste l'enjeu majeur de la candidature de l'équitation de tradition française à l'inscription au patrimoine culturel immatériel de l'UNESCO.

Enfin et surtout - on ne le répétera jamais assez -, le cheval relève d'un patrimoine vivant. Il faut entendre par là, en premier lieu, des « races » créées et maintenues par l'homme, dont la conservation incombait autrefois aux Haras nationaux et à leurs dépôts d'étalons, et dont plusieurs sont actuellement en voie de disparition (boulonnais) ou en déclin démographique (anglo-arabe). Il faut entendre, en second lieu, une riche médecine vétérinaire dont les racines sont à la fois équines et françaises (voir la communication de Christophe Degueurce), médecine à laquelle s'ajoute un art, plus ancien encore, de la maréchalerie (voir le fameux traité de Pierre-Étienne Lafosse de 1772). Il faut entendre en troisième lieu, les chevaux dressés - «mis », comme disent les cavaliers - qui, comme l'a montré Patrice Franchet d'Espèrey dans plusieurs de ses ouvrages ${ }^{1}$, doivent être considérés comme les dépositaires du savoir-faire des écuyers, et des transmetteurs de ce savoir-faire aux élèves-écuyers. Il faut entendre, enfin, les chevaux en tant que moteur vivant des véhicules hippomobiles. À la question de savoir pourquoi les voitures hippomobiles suscitaient moins d'engouement chez les collectionneurs que les automobiles anciennes, François Poplin faisait justement remarquer qu'il manquait aux premières leur moteur vivant (ainsi, sans doute, que des pièces de rechange, objets des réserves formulées ici même par Nathalie Giffard de La Jaille).

On ne saurait terminer ce (trop) rapide panorama sans évoquer, pour les déplorer, trois handicaps qui pèsent sur l'étude des patrimoines du cheval.

Le premier tient à la distinction entre "patrimoine culturel matériel » et "patrimoine culturel immatériel », comme si l'un pouvait se concevoir sans l'autre. Il faut espérer que cette distinction, véritable obstacle à la pensée, n'est qu'administrative et, donc, faite pour être contournée...

Un deuxième handicap tient à l'indifférence voire au mépris dans lequel les sciences de l'homme et de la société, dont l'histoire (à quelques exceptions remarquables près comme celle de Daniel Roche) tiennent l'objet cheval, indifférence ou mépris qui n'a d'égal que celui que nourrissent les professionnels et praticiens du cheval à l'endroit des sciences de l'homme et de la société .

13 Troisième handicap et troisième coupure: le fossé qui sépare le point de vue des historiens de l'art et celui des ethnologues. La position des premiers a été illustrée par Jean-Louis Libourel - position qu'il a lui-même qualifiée d'« un peu élitiste »- lorsque celui-ci s'est déclaré intéressé par les voitures de luxe, relevant de la carrosserie, à l'exclusion des véhicules utilitaires qui ne relèvent, eux, que du charronnage! Un autre historien de l'art, Jean-Michel Leniaud, s'est distingué en insistant au contraire sur l'importance des séries d'«objets ordinaires » et de la contribution des ethnologues au travail de description et de catalogage de tels objets (pour avoir entendu, au moment du transfert des collections du Musée de l'Homme au Musée du quai Branly, des historiens de l'art parler de ces collections comme de la «merde ethnographique ", j'avoue avoir particulièrement apprécié les propos de Jean-Michel Leniaud).

14 Et puisque l'ethnologue que je suis en est à restituer des propos entendus «sur le terrain ", je voudrais terminer en évoquant les réactions, entre incrédulité et dérision, 
qui échappaient à certains interlocuteurs devant lesquels j'évoquais, il y a quelques semaines, l'opération «Le cheval et ses patrimoines»: "Ah! Le cheval, vraiment? Qu'est-ce que cela a donc de si important?». Cette incompréhension manifeste justifie que soit peaufiné notre argumentaire. L'omniprésence du cheval jusque dans les années 1950 et les nombreuses traces que celle-ci a entraîné dans presque tous les domaines est bien sûr fondamentale : l'attitude qui consisterait à occulter le rôle et la place du cheval dans l'histoire équivaudrait à celle d'un historien du XXe siècle qui ferait l'impasse sur l'automobile. Mais il y a plus ou autre chose. Aujourd'hui, un nouvel engouement se manifeste, qui tire le cheval vers un statut proche de celui d'un animal de compagnie. De cette évolution, dont on conviendra qu'elle n'est pas sans danger pour l'équidé et pour son statut, heureusement encore agricole, le cheval est relativement protégé par sa taille, par sa puissance, par son influx qui en font un animal pouvant être dangereux. Autrement dit, le cheval serait, à côté du chat et du chien, le seul animal de compagnie avec lequel on ne peut pas faire n'importe quoi. Après avoir grandi et fortifié l'homme - à cheval, l'espace s'élargit (pensons à la "perspective cavalière" des artistes), le temps s'accélère (avancer sur un cheval au galop impose de penser plus vite qu'à pied) -, après lui avoir offert, selon le mot de Francis Ponge, « un trône à sa mesure ", le cheval pourrait aujourd'hui rendre à l'homme le service de lui remettre les pieds sur terre et de le ramener ainsi à certaines réalités. Oui, décidément, le cheval est un civilisateur de l'homme. À ce titre comme à d'autres, il justifie pleinement l'opération patrimoniale qui lui est consacrée par le ministère de la Culture, à laquelle nous participons aujourd'hui et à laquelle, je l'espère, nous participerons encore, ensemble, durant les deux prochaines années.

\section{NOTES}

1. - Voir notamment: FRANCHET D'ESPEREY, Patrice. La Main du maître. Réflexions sur l'héritage équestre. Paris : Odile Jacob, 2007.

2. - Voir DIGARD, Jean-Pierre. «Qu'ont à voir les sciences sociales avec le cheval ?». Le Mouvement Social, éditions La Découverte, n²29, octobre-décembre 2009, p. 3-11.

\section{AUTEUR}

\section{JEAN-PIERRE DIGARD}

directeur de recherche émérite au CNRS, Paris digard@ivry.cnrs.fr 